\title{
Non-perturbative improvement of bilinears in unquenched QCD *
}

\author{
T. Bhattacharya ${ }^{\mathrm{a}}$, R. Gupta ${ }^{a}$, W. Lee ${ }^{a}$ and S. Sharpe ${ }^{\mathrm{b}} \dagger$ \\ ${ }^{a}$ Los Alamos National Lab, MS B-285, Los Alamos, New Mexico 87545, USA \\ bPhysics Department, Box 351560, University of Washington, Seattle, WA 98195-1560, USA
}

We describe how the improvement of quark bilinears generalizes from quenched to unquenched QCD, and discuss which of the additional improvement constants can be determined using Ward Identities.

A major motivation for undertaking the improvement program is to facilitate unquenched calculations by allowing simulations at larger lattice spacings. Thus it is important to study the application of the improvement program to unquenched theories. This has been done for the action itself, and for certain operators in the chiral limit [1,2]. Here we take a further step by considering the theory of on-shell improvement of quark bilinears in unquenched QCD with nonzero quark masses. We enumerate the additional improvement coefficients that are required, and discuss which of them can be determined nonperturbatively using Ward Identities (WI).

The analysis depends on the number of dynamical flavors, and we consider here the physically relevant theories with $N_{f} \geq 3$ non-degenerate flavors. The two flavor theory is more complicated and will be discussed elsewhere [3]. We use an abbreviated notation for flavor traces

$$
\left\langle A_{\mu}\right\rangle=\operatorname{Tr}\left(A_{\mu}\right)=\sum_{j=1, N_{f}} A_{\mu}^{(j j)},\left\langle\lambda A_{\mu}\right\rangle=\operatorname{Tr}\left(\lambda A_{\mu}\right),
$$

with $\lambda$ an $S U\left(N_{f}\right)$ generator. Note that we consider both flavor singlets and non-singlets, with the latter being both off-diagonal and diagonal. All three are needed for phenomenology. For example, the flavor off-diagonal operator $A_{\mu}^{(23)}=$ $\bar{d} \gamma_{\mu} \gamma_{5} s$ is needed to determine $f_{K}$, and the nucleon matrix elements of the flavor diagonal operators $A_{\mu}^{j j}$ and $T_{\mu \nu}^{j j}$ (which, with $j$ an unsummed flavor index, are linear combinations of flavor

\footnotetext{
*Supported by DOE contracts DE-FG03-96ER40956 and DOE-W7405-ENG-86.

${ }^{\dagger}$ Speaker
}

singlet and non-singlet) give information on the structure functions.

We begin by reviewing previous work on nonperturbative $O(a)$ improvement of unquenched QCD. The ALPHA collaboration [1] has shown how on-shell improvement of the action can be accomplished by adding the SheikholeslamiWohlert or "clover" term, with appropriately chosen coefficient $c_{S W}$. On-shell matrix elements of flavor non-singlet axial, vector and tensor bilinears are then improved, in the chiral limit, by adding dimension four operators:

$$
\begin{aligned}
\left\langle\lambda A_{\mu}\right\rangle^{I} & =\left\langle\lambda A_{\mu}\right\rangle+c_{A} \partial_{\mu}\langle\lambda P\rangle \\
\left\langle\lambda V_{\mu}\right\rangle^{I} & =\left\langle\lambda V_{\mu}\right\rangle+c_{V} \partial_{\nu}\left\langle\lambda T_{\mu \nu}\right\rangle \\
\left\langle\lambda T_{\mu \nu}\right\rangle^{I} & =\left\langle\lambda T_{\mu \nu}\right\rangle+c_{T}\left[\partial_{\mu}\left\langle\lambda V_{\nu}\right\rangle-\partial_{\nu}\left\langle\lambda V_{\mu}\right\rangle\right]
\end{aligned}
$$

Here we use the standard notation for local lattice bilinears (see [1, 他), and, for brevity, set the lattice spacing $a$ to unity. Flavor non-singlet scalar and pseudoscalar bilinears are automatically onshell $O(a)$ improved in the chiral limit.

The improvement coefficients $c_{S W}$ and $c_{\Gamma}(\Gamma=$ $V, A, T)$ depend on the number of dynamical quarks, $N_{f}$, and on the bare coupling $g_{0}^{2}$. They can all be determined non-perturbatively using WI. In particular, enforcing the partial conservation of the improved flavor off-diagonal axial current determines $c_{S W}$ and $c_{A}$, as well as the critical quark mass $m_{c}$ at which chiral symmetry is restored [1]. This method works for two or more flavors, and has been implemented numerically for $N_{f}=2$ [2]. With the improved axial current in hand, one can then enforce the axial transformation properties of the vector and ten- 
sor bilinears to determine $c_{V}$ [5, 4 , and $c_{T}[6]$.

Away from the chiral limit there are many additional improvement coefficients. In particular, the effective coupling constant becomes [1]

$g_{\mathrm{eff}}^{2}=g_{0}^{2}\left(1+b_{g}\langle M\rangle / N_{f}\right)$,

where $\langle M\rangle$ is the trace of the mass matrix. Knowledge of the improvement coefficient $b_{g}$ allows one to adjusts $g_{0}$, as $\langle M\rangle$ is varied, such that $g_{\text {eff }}$ is constant. This corresponds to working at fixed lattice spacing. In this way one does not introduce spurious $O(a M)$ dependence in physical quantities. Methods for determining $b_{g}$ nonperturbatively are given in Refs. [1] 6 ].

This completes our review of previous work. We now describe the new improvement coefficients that arise when one improves bilinears in the unquenched theory. First we note that, in the chiral limit, improvement of flavor singlet bilinears is more complicated than that of nonsinglets, due to the contributions from disconnected contractions. For axial and vector currents the form remains the same, e.g.

$\left\langle A_{\mu}\right\rangle^{I}=\left\langle A_{\mu}\right\rangle+\bar{c}_{A} \partial_{\mu}\langle P\rangle$,

with new constants $\bar{c}_{A}$ and $\bar{c}_{V}$. For the other bilinears there are additional gluonic operators

$$
\begin{aligned}
\langle S\rangle^{I}= & \langle S\rangle+g_{S} \operatorname{tr}\left(F_{\mu \nu} F_{\mu \nu}\right) \\
\langle P\rangle^{I}= & \langle P\rangle+g_{P} \operatorname{tr}\left(F_{\mu \nu} \tilde{F}_{\mu \nu}\right) \\
\left\langle T_{\mu \nu}\right\rangle^{I}= & \left\langle T_{\mu \nu}\right\rangle+\bar{c}_{T}\left[\partial_{\mu}\left\langle V_{\nu}\right\rangle-\partial_{\nu}\left\langle V_{\mu}\right\rangle\right] \\
& \quad+g_{T}\left[\operatorname{tr}\left(F_{\rho \mu} F_{\rho \nu}\right)-\operatorname{tr}\left(F_{\rho \nu} F_{\rho \mu}\right)\right] .
\end{aligned}
$$

In principle, one can determine all six new improvement coefficients by enforcing the invariance of these operators $O^{I}$ under an off-diagonal vector transformation, i.e. $\delta_{V}^{(12)} O^{I}=0$. This equation can be made non-trivial by taking appropriate flavor non-singlet matrix elements.

Moving away from the chiral limit, there are several new improvement terms. For flavor nonsinglets, the general form is exemplified by

$$
\begin{aligned}
& \left\langle\widehat{\lambda A_{\mu}}\right\rangle=Z_{A}\left[\left(1+\bar{b}_{A}\langle M\rangle\right)\left\langle\lambda A_{\mu}\right\rangle^{I}\right. \\
& \left.\quad+b_{A} \frac{1}{2}\left\langle\{\lambda, M\} A_{\mu}\right\rangle+f_{A}\langle\lambda M\rangle\left\langle A_{\mu}\right\rangle\right],
\end{aligned}
$$

$\overline{{ }^{3} \text { Normalized such that }} S_{\text {glue }}=\left(1 / 2 g_{0}^{2}\right) \operatorname{tr}\left(F_{\mu \nu} F_{\mu \nu}\right)$. where the "hat" indicates an improved and renormalized operator. CP invariance implies that only the anticommutator of $\lambda$ and $M$ appears. It is instructive to consider examples of this general formula. For flavor off-diagonal operators, the $f$-term drops out, leaving

$\widehat{\left.A_{\mu}^{(12}\right)}=Z_{A}\left(A_{\mu}^{(12)}\right)^{I}\left[1+\bar{b}_{A}\langle M\rangle+b_{A} \frac{1}{2}\left(m_{1}+m_{2}\right)\right]$

The $b_{A}$ term is that present in the quenched approximation, while $\bar{b}_{A}$ multiplies the additional mass dependence arising from quark loops. All terms contribute for diagonal operators, e.g.

$$
\begin{aligned}
& \widehat{A_{\mu}^{(11)}}-\widehat{A_{\mu}^{(22)}}=Z_{A}\left[\left(A_{\mu}^{(11)}-A_{\mu}^{(22)}\right)^{I}\left(1+\bar{b}_{A}\langle M\rangle\right)\right. \\
& \left.\quad+b_{A}\left(m_{1} A_{\mu}^{(11)}-m_{2} A_{\mu}^{(22)}\right)+f_{A}\left(m_{1}-m_{2}\right)\left\langle A_{\mu}\right\rangle\right]
\end{aligned}
$$

The $f$-term arises from disconnected contractions of the operator, and is present only for nondegenerate quarks.

Finally, we consider the mass-dependent improvement coefficients needed for flavor singlet operators. Here there are only two independent traces, and thus two terms per bilinear, e.g.

$$
\widehat{\left\langle A_{\mu}\right\rangle}=Z_{A} r_{A}\left[\left(1+\bar{d}_{A}\langle M\rangle\right)\left\langle A_{\mu}\right\rangle^{I}+d_{A}\left\langle M A_{\mu}\right\rangle\right]
$$

Note that the overall normalization constant differs from $Z_{A}$ by a factor $r_{A}$. For the axial current, this factor is scale-dependent since the singlet current has a non-zero anomalous dimension. For the other bilinears, however, $r_{\Gamma}$ is a finite, scaleindependent quantity, dependent only on the effective coupling constant and on $N_{f}$. This is because the anomalous dimensions of the singlet and non-singlet bilinears are the same.

In summary, the number of improvement terms involving mass dependence increases substantially when one unquenches and considers both singlet and non-singlet bilinears. Improvement of flavor off-diagonal operators in the quenched theory requires the determination of 5 such coefficients, while complete improvement of bilinears in the unquenched theory requires 25 . In the quenched theory, all 5 coefficients can be determined using WI with non-degenerate quarks [4]. We have studied the generalization of this method to unquenched QCD, and find that all but three 
of the new coefficients can be determined using WI with the following steps. I

1. Enforcing the conservation of the diagonal vector charges while independently varying the three quark masses determines all five coefficients associated with the vector bilinear (as well as $r_{V}$ ).

2. Enforcing the PCAC relation away from the chiral limit (combined with considerations outlined below) determines the combinations $b_{S}$, $b_{P}-b_{A}, \bar{b}_{P}-\bar{b}_{A}-\bar{b}_{S}, f_{S}, d_{S}-3 \bar{b}_{S}$, and $\bar{d}_{S}-\bar{b}_{S}$, as well as $r_{S}$. This generalizes the method of Ref. [7]. 3. Enforcing the off-diagonal axial transformation properties of operators, for non-degenerate quark masses. This is the generalization of the method of Ref. 肚, and determines $b_{V}+b_{A}, \bar{b}_{V}+\bar{b}_{A}, b_{P}+$ $b_{S}, 2 \sqrt{r_{S}}\left(\bar{b}_{P}-\bar{b}_{S}\right)+b_{P}-b_{S}$, and $b_{T}$.

4. Enforcing off-diagonal vector transformation properties of operators, e.g.

$\delta_{V}^{(12)} O^{(21)}=O^{(11)}-O^{(22)}, \quad \delta_{V}^{(12)}\langle O\rangle=0$.

These identities have not been considered previously, since they involve flavor diagonal bilinears. They determine the $f_{\Gamma}(\Gamma=V, A, T, S, P)$ together with $d_{A}, d_{P}$ and $d_{T}$, and $r_{P}$.

5. Enforcing the flavor off-diagonal axial transformations of flavor singlet operators, e.g. $\delta_{A}^{(12)}\langle P\rangle=S^{(12)}$. These determine $\bar{d}_{P}-\bar{b}_{S}$, $\bar{d}_{S}-\bar{b}_{P}, \bar{d}_{T}-\bar{b}_{T}$, and $r_{T}$.

The remaining undetermined combinations are $\bar{b}_{S}+\bar{b}_{P}+\bar{d}_{S}+\bar{d}_{P}, \bar{b}_{T}+\bar{d}_{T}$ and $\bar{d}_{A}\left(\right.$ and $\left.r_{A}\right)$.

We close by expanding upon one feature of our analysis. In the second step, when we enforce the PCAC relation, we need the $O(a)$ improved expression for quark masses

$$
\begin{aligned}
& \langle\widehat{\lambda M}\rangle=Z_{m}\left[\langle\lambda M\rangle\left(1+\bar{b}_{m}\langle M\rangle\right)+b_{m}\left\langle\lambda M^{2}\right\rangle\right] \\
& \widehat{\langle M\rangle}=Z_{m} r_{m}\left[\langle M\rangle\left(1+\bar{d}_{m}\langle M\rangle\right)+d_{m}\left\langle M^{2}\right\rangle\right] .
\end{aligned}
$$

Note that singlet and non-singlet masses are normalized differently, so that individual masses have off-diagonal renormalization even at $O(1)$ :

$$
\widehat{m}_{j}=Z_{m}\left[m_{j}+\left(r_{m}-1\right)\langle M\rangle / N_{f}+O(a)\right] .
$$

This effect is absent in the quenched approximation, where $r_{m}=1$, as well as $d_{m}=b_{m}$, $\bar{b}_{m}=\bar{d}_{m}=0$.

\footnotetext{
${ }^{4}$ Some of combinations of coefficients depend on $N_{f}$, and we quote results here for $N_{f}=3$.
}

It turns out that these new improvement coefficients are related to those we have previously introduced, and we need to determine these relationships in order to extract all relevant information in step 2 above. In other words, we need to generalize the quenched relations $Z_{m} Z_{S}=1$ and $b_{S}=-2 b_{m}$ [8]. To do so we note that it is consistent with the WI to take the matrix elements of the improved renormalized scalar density to be

$\left\langle H\left|\widehat{S^{(j j)}}\right| H\right\rangle=\left.\frac{\partial m_{H}}{\partial \widehat{m}_{j}}\right|_{\widehat{m}_{k \neq j}, a}$,

with $H$ an arbitrary hadronic state. Note that the lattice spacing, and not $g_{0}^{2}$, is held fixed in the derivative. The r.h.s. can be evaluated in terms of derivatives known from the form of the lattice action

$$
\begin{aligned}
\left.\frac{\partial m_{H}}{\partial m_{j}}\right|_{m_{k \neq j}, g_{0}} & =\left\langle H\left|S^{(j j)}\right| H\right\rangle, \\
-\left.2 g_{0}^{4} \frac{\partial m_{H}}{\partial g_{0}^{2}}\right|_{m_{j}} & =\left\langle H\left|\operatorname{tr}\left(F_{\mu \nu} F_{\mu \nu}\right)\right| H\right\rangle .
\end{aligned}
$$

This results in the relations ${ }^{4} Z_{S} Z_{m}=1, r_{S} r_{m}=$ $1, b_{S}=-2 b_{m}, \bar{b}_{S}=-\bar{b}_{m}, 3 f_{S}=2\left(b_{m}-d_{m}\right)$, and $3 \bar{d}_{S}=3 \bar{b}_{m}+2 b_{m}-6 \bar{d}_{m}-2 d_{m}$. One also finds two constraints, namely $d_{S}=3 \bar{b}_{S}+b_{S}$, and $2 g_{S}=$ $b_{g} / g_{0}^{2}$. The latter can be used as a consistency check on the calculations of $g_{S}$ and $b_{g}$.

\section{REFERENCES}

1. K. Jansen et al., Phys. Lett. B372 (1996) 275; M. Lüscher et al., Nucl. Phys. B478 (1996) 365.

2. K. Jansen and R. Sommer, Nucl. Phys. B530 (1998) 185.

3. T. Bhattacharya et al, in preparation.

4. T. Bhattacharya et al, hep-lat/9904011.

5. M. Guagnelli and R. Sommer, Nucl. Phys. B(Proc. Suppl.)63 (1998) 886.

6. G. Martinelli et al., Phys. Lett. B411 (1997) 141.

7. G.M. de Divitiis et al., Phys. Lett. B419 (1998) 311.

8. S. Sint and P. Weisz, Nucl. Phys. B(Proc. Suppl.)63 (1998) 856. 